\title{
Genetic diversity of Yersinia pestis in Brazil
}

\author{
M.B.M. Oliveira', M.P.S. Barros ${ }^{2,3}$, V.M. Silveira-Filho ${ }^{2,3}$, \\ M.R. Araújo-Nepomuceno ${ }^{2}$, V.Q. Balbino ${ }^{3}$, N.C. Leal' ${ }^{2}$, \\ A.M.P. Almeida ${ }^{2}$ and T.C. Leal-Balbino ${ }^{2,3}$ \\ ${ }^{1}$ Departamento de Bioquímica, Universidade Federal de Pernambuco, \\ Recife, PE, Brasil \\ ${ }^{2}$ Centro de Pesquisas Ageu Magalhães (CPqAM/FIOCRUZ-PE), \\ Recife, PE, Brasil \\ ${ }^{3}$ Departamento de Genética, Universidade Federal de Pernambuco, \\ Recife, PE, Brasil \\ Corresponding author: T.C. Leal-Balbino \\ E-mail: cristina@cpqam.fiocruz.br / tcristinaleal@yahoo.com.br
}

Genet. Mol. Res. 11 (3): 3414-3424 (2012)

Received November 8, 2011

Accepted April 11, 2012

Published September 25, 2012

DOI http://dx.doi.org/10.4238/2012.September.25.10

\begin{abstract}
Plague outbreaks are occasionally reported in Brazil. Unfortunately, due to great genetic similarity, molecular subtyping of Yersinia pestis strains is difficult. Analysis of multiple-locus variable number of tandem repeats (VNTR), also known as MLVA, has been found to be a valuable tool to discriminate among strains. To check for genetic differences, strains obtained from two different ecological complexes in Brazil collected during two different epidemiological events, an epizootic in Sítio Alagoinha in 1967 and an outbreak in Planalto da Borborema in 1986, were subtyped through MLVA using 12 VNTR loci. Three clusters (A, B and C) were observed. Of the 20 strains from the epizootic, 18 fit into cluster A. Cluster A was divided into two subgroups: $A_{1}$ (15 strains) and $A_{2}$ ( 3 strains). Of the 17 strains from the outbreak, 15 fit into cluster B. Cluster B was divided into three subgroups: $\mathrm{B}_{1}$ (4 strains), $\mathrm{B}_{2}$ (4 strains) and $\mathrm{B}_{3}$ (7 strains). Cluster $\mathrm{C}$ is a singleton with one epizootic strain. The external standards, $Y$. pestis CO92 and Y. pseudotuberculosis IP32953, formed two clusters of singletons. The stability of 12 VNTR loci of three unrelated cultures
\end{abstract}


included in this study was assessed. The 12 VNTR loci were stable through multiple serial subcultures in the laboratory. MLVA revealed that $Y$. pestis populations in Brazil are not monomorphic, and that there is intraspecific genetic diversity among Brazilian plague strains. We conclude that there is some correlation among genetic groups of this species, related to the temporal and geographic origin of isolates.

Key words: Plague; Molecular epidemiology; MLVA; Yersinia pestis

\section{INTRODUCTION}

The plague, caused by infection with Yersinia pestis, was introduced in Brazil by sea through infected rats and fleas carried on steam ships during the last pandemic, which originated in China in 1855. Records of the early history of the plague in Brazil are sporadic and sparse, which makes detailed tracking difficult to impossible. Systematic information became available only after the 1930s (Pollitzer, 1954; Baltazard, 1968). Nevertheless, there is a general consensus that the first human case was registered in October 1899, in Santos, São Paulo State. From then on, other coastal cities became successively infected. Government control measures were promptly taken, and the infection was eliminated from the coast; however, these measures did not stop the spread of the plague to inland cities, towns and villages. The plague reached rural areas, afflicting small districts, farms and ranches. The plague then established many natural foci among the wild rodents in several ecological complexes, from the State of Ceará to the northern Minas Gerais (collectively called the Northeast focus) and in the Serra dos Orgãos, Rio de Janeiro (WHO, 1965; Baltazard, 1968).

Tight control of the plague in the 1930s reduced human cases and mortality. Unfortunately, this program was interrupted, and the disease reemerged in the 1960s. To achieve new insights into the features of plague epidemiology and to reorganize an effective control strategy, an extensive project comprised of field and laboratory research was established and carried out from 1966 to 1974 (Baltazard, 2004). By the end of the project, more than 600 Y. pestis strains were isolated, which resulted in a unique collection of $Y$. pestis cultures in Brazil. Subsequent epidemiological investigations and surveillance activities in northeastern Brazilian plague areas expanded this collection (Rocha et al., 2009). The collection is now maintained by the National Service for Reference in Plague of the Centro de Pesquisas Aggeu Magalhães (SRP/ CPqAM), Recife, PE, and linked to FIOCRUZ culture collections (Fiocruz-CYP).

Attempts have been made to genotype many of these isolates. In almost every case, no differences between isolates were found, even across multiple host species, different geographic origins, or years (Sobreira M and Leal NC, unpublished results). Molecular subtyping of Y. pestis for epidemiological tracking has been difficult due to the great genetic similarity among isolates (Vogler et al., 2011). However, analysis of multiple-locus variable number of tandem repeats (VNTR), also known as MLVA, has become a valuable tool to discriminate $Y$. pestis strains. Using MLVA, it is possible to trace the dispersion routes of bacterial populations at the local, regional and global levels (Adair et al., 2000; Klevytska et al., 2001; Achtman et al., 2004; Girard et al., 2004; Pourcel et al., 2004; Lowell et al., 2005; Vogler et al., 2011).

In the present study, we analyzed Y. pestis strains from two different ecological com- 
plexes in Brazil during two different epidemiological events. MLVA using 12 VNTRs revealed that $Y$. pestis populations in Brazil are not monomorphic. We also demonstrated intraspecific genetic diversity among the strains, which establishes a relative correlation between genetic groups and the temporal and geographic origin of the isolates.

\section{MATERIAL AND METHODS}

\section{Bacterial strains studied}

The study involved 37 Y. pestis strains that were kindly provided by the Fiocruz-CYP culture collection, which is maintained by the SRP/CPqAM-FIOCRUZ/PE. Twenty strains were isolated from rodents and fleas during an epizootic in August 1967. This epizootic occurred in Sítio Alagoinha, a locality situated in Chapada do Araripe, Pernambuco State (de Almeida et al., 1985). Seventeen strains were isolated from an outbreak in 1986, which involved several municipalities in Planalto da Borborema, Paraíba State (de Almeida et al., 1989). Three unrelated strains (P. CE 882, P. Exu 340 and P. Peru 375) and 11 derived cultures obtained through multiple serial subcultures of the parental strains (Leal-Balbino et al., 2004) were included to assess in vitro VNTR stability. Figure 1 shows the localization of the Brazilian plague foci and origin of the strains.

Epizootic strains were identified as P (plague), Exu (laboratory city), and sequential number. Outbreak strains were identified as P (plague), PB (state of origin) and sequential number.

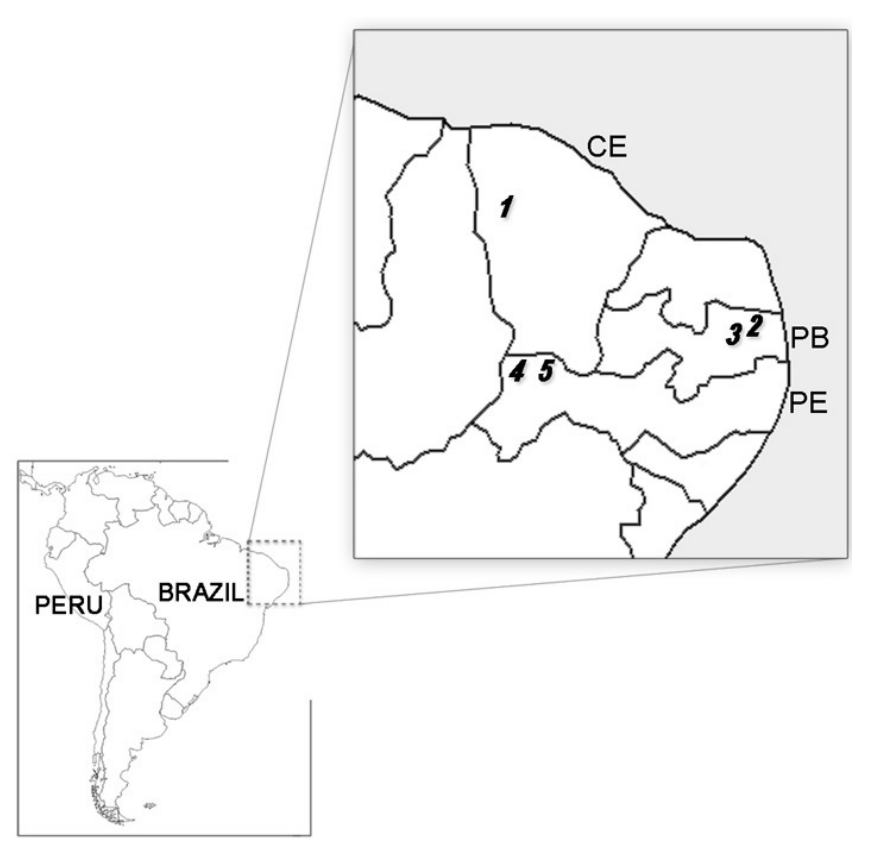

Figure 1. Geographic origin of the Yersinia pestis strains analyzed. CE = Ceará (1: Ipu); PB = Paraíba (2: Solânea, 3: Remígio), and PE = Pernambuco (4: Ipubi, 5: Exu). 


\section{Culture conditions}

Cultures were maintained on peptone agar stabs at $4^{\circ} \mathrm{C}$. The strains were inoculated into brain heart infusion broth at $28^{\circ} \mathrm{C}$ and inspected daily for up to seven days. Each culture grown during this period was plated on blood agar base, incubated at $28^{\circ} \mathrm{C}$ and analyzed on a daily basis for up to five days to assess the morphology of the developing colonies. Identification of the culture and confirmation of purity were carried out by the plague phage test (Karimi, 1978).

\section{VNTR primers}

Primers designed to amplify the 12 chromosomal VNTRs published by Adair et al. (2000), Le Flèche et al. (2001) and Klevytska et al. (2001) were used for PCR amplification in the $Y$. pestis cultures. It should be pointed out that some loci were given different names by different authors. In this paper, the standardized nomenclature from Pourcel et al. (2004) was used. A description of the primers and references is presented in Table 1.

Table 1. Sequences and description of the primers used to analyze the 12 VNTR loci in Yersinia pestis strains.

\begin{tabular}{|c|c|c|c|c|}
\hline Locus* & Alias & Primer sequence & Repeat unit (bp) & Reference for primer \\
\hline \multirow[t]{2}{*}{$\mathrm{ms} 04$} & M58 & F: 5'-GCG ATA ACC CAC ATT ATC ACA ATA ACC AAC AC-3' & 17 & Klevytska et al. (2001) \\
\hline & & R: 5'-GCT GAT GGA ACC GGT ATG CTG AAT TTG C-3' & & \\
\hline \multirow[t]{2}{*}{$\mathrm{ms} 05$} & M59 & F: 5'-GCT TAG CCG CCA GAA AAG GTG AGT TGG C-3' & 17 & Klevytska et al. (2001) \\
\hline & & R: 5'-GAT AAT GGC GGT AGC CGG AAT CTG ATA ATC ATC-3' & & \\
\hline \multirow[t]{2}{*}{ ms06 } & - & F: 5'-AAT TTT GCT CCC CAA ATA GCA T-3' & 60 & Le Flèche et al. (2001) \\
\hline & & R: 5'-TTT TCC CCA TTA GCG AAA TAA GTA-3' & & \\
\hline \multirow[t]{2}{*}{ ms07 } & M37 & F: 5'-GCC ACA GGA AGA GGA CAT TTC AGA GAA AAC-3' & 10 & Klevytska et al. (2001) \\
\hline & & R: 5'-GTT GCT AAA ACG ATA CCG CTA CGA TCA GC-3' & & \\
\hline \multirow[t]{2}{*}{$\mathrm{ms} 09$} & - & F: 5'-CGT TAC CCT TGT TGC CAA TAG T-3' & 18 & Le Flèche et al. (2001) \\
\hline & & R: 5'-ACG CAG AAC ATG CTT ACC TTT TAT-3' & & \\
\hline \multirow[t]{2}{*}{$\mathrm{ms} 20$} & M51 & F: 5'-GCA ACC CGC TGA AGT TGT AAA AAC CGA C-3' & 15 & Klevytska et al. (2001) \\
\hline & & R: 5'-GCG TTG ATC TTC GCG GCC TTC AC-3' & & \\
\hline \multirow[t]{2}{*}{ ms30 } & - & F: 5'-CAA TAA TAC CAT CGT GCG TGA TAC-3' & 54 & Le Flèche et al. (2001) \\
\hline & & R: 5'-TAT TAA TGG TGG TGT TAG TCG CTG T-3' & & \\
\hline \multirow[t]{2}{*}{ ms45 } & M42 & F: 5'-GCC GGT AGA GGC GTT GTC TTT GGT TTT TTC-3' & 12 & Klevytska et al. (2001) \\
\hline & & R: 5'-GTT TTG GGG TTC AGT GCA CGC TTG TGA C-3' & & \\
\hline \multirow[t]{2}{*}{ ms46 } & - & F: 5'-CAG GTT TTA CGT TAT TTT CTG AAG G-3' & 7 & Le Flèche et al. (2001) \\
\hline & & R: 5'-CAG CAT GAA GTA TGA CGG GTA TAT TA-3' & & \\
\hline \multirow[t]{2}{*}{ ms54 } & - & F: 5'-GTC CAC CAT TTT CAT ACT GTC ACT T-3' & 22 & Le Flèche et al. (2001) \\
\hline & & R: 5'-GCT CTT TGT TCG ATT TTA TTG AAT G-3' & & \\
\hline \multirow[t]{2}{*}{ ms62 } & M34 & F: 5'-GAA TCG CGG GTT GAC GCT GTT GAG C-3' & 9 & Klevytska et al. (2001) \\
\hline & & R: 5'-GCT GAA CAG CCC CAT AAA ACC GGA GC-3' & & \\
\hline \multirow[t]{2}{*}{$1 \mathrm{AB}$} & - & F: 5'-GGT TAG GTA GGG TGT TGA AG-3' & 4 & Adair et al. (2000) \\
\hline & & R: 5'-AAA GAG GCT AAG TGG CAA-3' & & \\
\hline
\end{tabular}

*Nomenclature as outlined in Pourcel et al. (2004).

\section{DNA extraction and PCR amplification}

Genomic DNA was prepared as described by Souza et al. (2007). The PCRs were carried out in a final volume of $25 \mu \mathrm{L}$ that contained $20 \mathrm{ng}$ DNA, 20 pmol of each primer, 50 mmol KCl, 20 mmol Tris-HCl, pH 8.0, $0.16 \mathrm{mmol}$ deoxynucleoside triphosphates (dNTPs, Invitrogen, Brazil), $1.5 \mathrm{mmol} \mathrm{MgCl}_{2}$, and $1 \mathrm{U}$ Taq DNA polymerase (Invitrogen).

The amplification of locus $1 \mathrm{AB}$ was carried out as described by Adair et al. (2000). The amplification of the other loci was carried out according to Le Flèche et al. (2001). The 
amplicons were separated by electrophoresis with 3.0\% agarose gels in Tris-borate at $100 \mathrm{~V}$ and were stained with $1 \mu \mathrm{g} / \mathrm{mL}$ ethidium bromide. The images were captured under UV using the 1-D Image Analysis version 3.5 software (Kodak Digital Science, New Haven, CT, USA). A 50-bp DNA ladder (Invitrogen) was used as a standard.

\section{Cluster analysis}

The 1-D Image Analysis imaging software (version 3.5; Kodak Digital Science) was used to determine the size of the amplicons. The number of repeat units (NRU) for each locus was determined using the following formula, which we inferred from Adair et al. (2000):

$$
N R U=A_{S}-F R_{S} / R U_{S}
$$

where $A_{S}$ is the amplicon size, $F R_{S}$ is the size of the flanking region sequence and $R U_{S}$ is the size of each repeat unit. $\mathrm{FR}_{\mathrm{S}}$ and $\mathrm{RU}_{\mathrm{S}}$ were identified by in silico analysis of the $Y$. pestis $\mathrm{CO} 92$ genome sequence with the Tandem Repeats Finder software (Benson, 1999). The Li et al. (2009) standardized nomenclature was used for all loci, except for ms30 (Le Flèche et al., 2001) and 1AB (Adair et al., 2000).

One representative amplicon of each VNTR was sequenced to confirm the size of the repeat and the copy number. Due to their small size (4 bp), all the amplicons generated with primer $1 \mathrm{AB}$ were sequenced to confirm the repeat size and copy number. For clustering analyses, the categorical coefficient and unweighted pair group method using arithmetic averages (UPGMA) were used. The use of the categorical parameter assumes that the character states are unordered. The same weight is given to a large or a small number of differences in the number of repeats at each locus. The UPGMA tree was constructed using MEGA v. 5.0 (Tamura et al., 2011).

MLVA patterns were normalized using Y. pestis CO92 (GI:30407161) and Y. pseudotuberculosis IP32953 (GI:51594359) public genomic data as external standards.

\section{Discriminatory analysis}

The numerical discriminatory index $(D I)$ of each VNTR locus was evaluated using the Hunter and Gaston (1988) formula:

$$
D I=1-[1 / N(N-1)] \Sigma n j(n j-1),
$$

where $N$ is the total number of isolates in this population, and $n j$ is the number of isolates representing each type. This approach is based on the probability that two unrelated strains from the test population will be placed into different groups.

\section{Sequencing}

For sequencing, the amplicons were purified with the Purelink PCR purification kit (Invitrogen), following manufacturer instructions. The sequencing reaction was performed with the same primers used for PCR. An ABI 3100 automated DNA sequencer (Applied Biosystems) and the BigDye kit (Applied Biosystems) were used for sequencing. The Basic Local Alignment Search Tool (BLASTN) (Altschul et al., 1997) was used to confirm the identity of the sequenced amplicons by comparing them against the NCBI databank of non-redundant nucleotide sequences. Seq Tools 8.3.022 (Rasmussen, 2002) and BioEdit v. 
7.0.5.2 (Hall, 1999) were used to edit the generated sequences.

The sequences were submitted to and published in the GenBank database under the following accession Nos. 1AB (GI:89112565); ms04 (GI:238867221); ms05 (GI:238867222); ms06 (GI:89112563); ms07 (GI:238867223); ms09 (GI:238867224); ms20 (GI:238867225); ms30 (GI:238867226); ms45 (GI:238867227); ms46 (GI:238867228); ms54 (GI:238867229), and ms62 (GI:238867230). The copy number variation of the VNTR loci was determined with the Tandem Repeats Finder tool (Benson, 1999) using the basic alignment parameters described on the homepage of the software (accessible at http://tandem.bu.edu/trf/trf.html).

\section{RESULTS}

MLVA based on 12 VNTR chromosomal loci was used to subtype two sets of $Y$. pestis strains from two different ecological complexes in Brazil during two different epidemiological events, an epizootic in Sítio Alagoinha in 1967 (20 strains) and an outbreak in Planalto da Borborema in 1986 (17 strains).

The primers directed to the 12 VNTRs yielded PCR products in all the cultures tested, which means that the loci were all present in the strains analyzed. MLVA was also performed on the 12 loci in the parental strain and their derivatives obtained by multiple serial subcultures of P. CE 882, P. Peru 375 and P. Exu 340 strains. Identical patterns were revealed in the parental and derived cultures (data not shown).

Of the 12 VNTR loci analyzed in the 37 outbreak and epizootic strains, seven (ms04, $\mathrm{ms} 05, \mathrm{~ms} 06$, ms $07, \mathrm{~ms} 20, \mathrm{~ms} 45$, and $\mathrm{ms} 62$ ) revealed polymorphisms. These seven loci displayed differences in amplicon length and copy number, with a $D I$ ranging from 0.4 to 0.9 . Strains from the two groups shared at least one allele in each locus. Five VNTRs revealed a monomorphic pattern: $\mathrm{ms} 09$ (20 repeats), ms30 (8), ms46 (4), ms54 (5), and 1AB (10) (Figures 2 and 3).

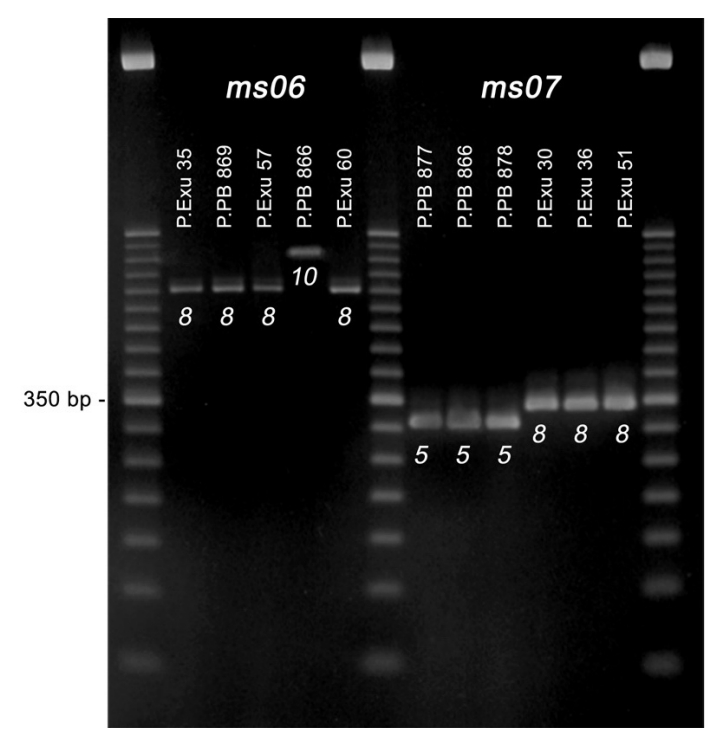

Figure 2. Analysis of PCR products by 3.0\% agarose gel electrophoresis to determine repeat size polymorphisms at the ms06 and ms07 loci. The number of repeats is indicated under each amplicon. 


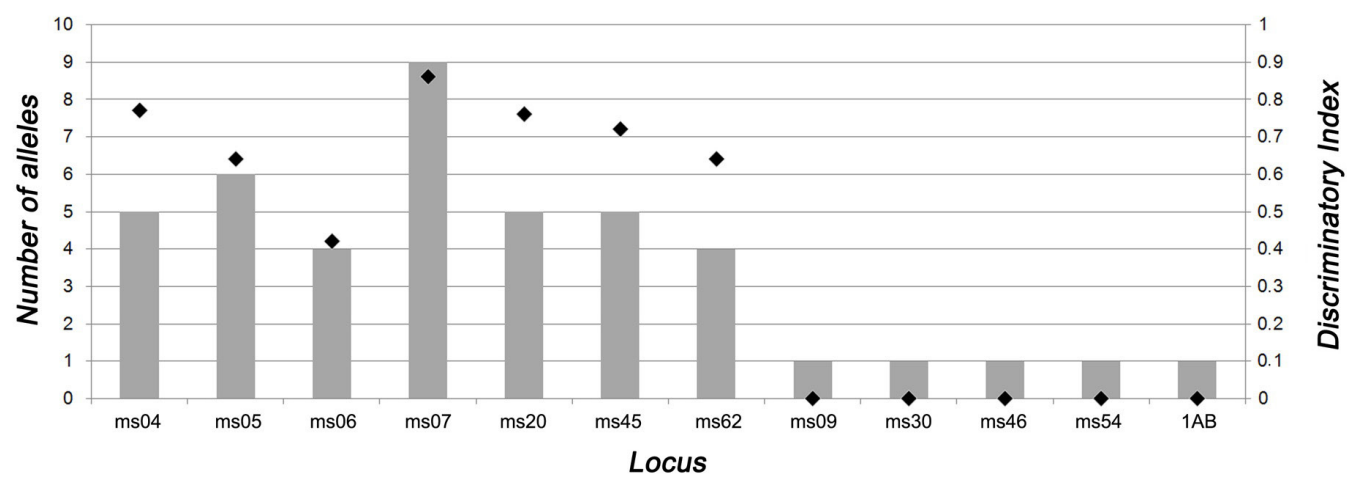

Figure 3. Distribution of the number of alleles (bars) and the discriminatory index (markers) found among the 12 VNTRs.

Using a cut-off value of 2.5 differences, the 37 epizootic and outbreak strains were separated into three distinct clusters (A, B and C). The external standards, Y. pestis CO92 and Y. pseudotuberculosis IP32953, formed two distinct singletons (Figure 4). Cluster A was divided into two subgroups: $A_{1}$ (15 strains) and $A_{2}$ (5 strains). Cluster $B$ was divided into three subgroups: $\mathrm{B}_{1}$ (4 strains), $\mathrm{B}_{2}$ (5 strains) and $\mathrm{B}_{3}$ (7 strains). Cluster $\mathrm{C}$ was a singleton (Figure 4 ).

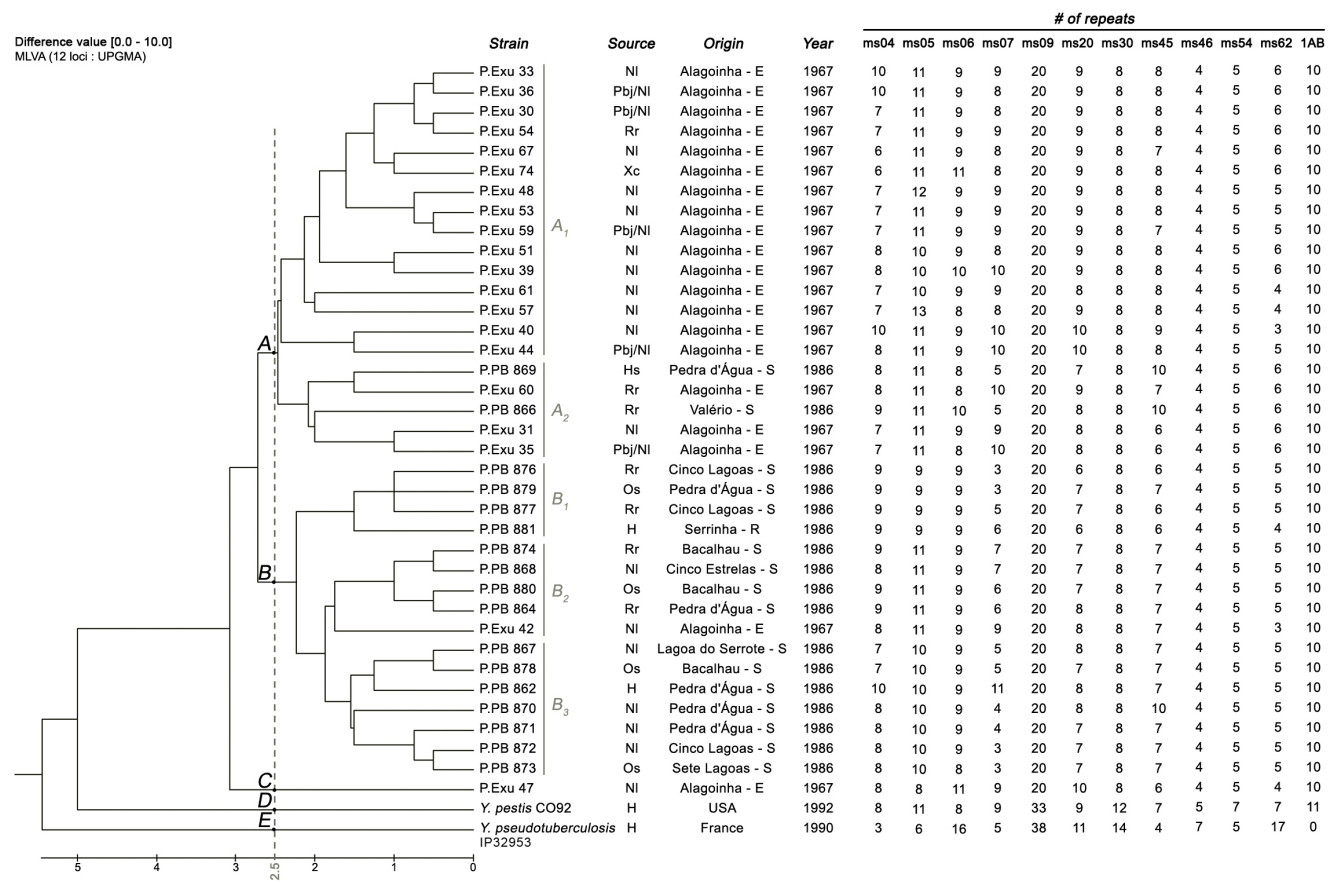

Figure 4. UPGMA dendrogram showing clustering and correlation of the Yersinia pestis strains studied with their origin and distribution by groups. $\mathrm{H}=$ Human; $\mathrm{Hs}=$ Holochilus sciureus; $\mathrm{Nl}=$ Necromys lasiurus; $\mathrm{Os}=$ Oryzomys subflavus $; \mathrm{Rr}=$ Rattus rattus; $\mathrm{Xc}=$ Xenopsylla cheopis $; \mathrm{Pbj} / \mathrm{Nl}=$ Polygenis bolshi jordani from $N$. lasiurus $; \mathrm{S}=$ Solânea; $\mathrm{E}=\mathrm{Exu} ; \mathrm{R}=$ Remígio; MLVA = multiple-locus variable number of tandem repeats. 
Cluster A, which was the dominant cluster, grouped all but two epizootic strains and included 2 outbreak strains (P. PB 866 and P. PB 869). Subgroup $A_{1}$ was specific for the epizootic strains and included most of them: Necromys lasiurus (9), Polygenis bolshi jordani (4), Rattus rattus (1), and Xenopsylla cheopis (1). Subgroup $\mathrm{A}_{2}$ included 3 epizootic strains: $N$. lasiurus (1), P. b. jordani (1), and $R$. rattus (1). Subgroup $\mathrm{A}_{2}$ also included 2 outbreak strains: Holochilus sciureus (1) and $R$. rattus (1) (Figure 4).

Cluster B included all but two outbreak strains plus one epizootic strain. Subgroups $B_{1}$ and $\mathrm{B}_{3}$ were exclusively composed of outbreak strains. Subgroup $\mathrm{B}_{1}$ included strains from $R$.

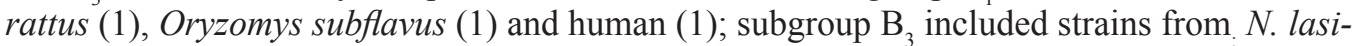
urus (4), O. subflavus (2) and human (1). Subgroup $\mathrm{B}_{2}$ included outbreak strains from $R$. rattus (2), N. lasiurus (1) and O. subflavus (1) and one epizootic strain from $N$. lasiurus (Figure 4).

Some strains from the wild host, the rodent $N$. lasiurus, and its flea vector, P. b. jordani, e.g., P. Exu 33/P. Exu 36, P. Exu 53/P. Exu 59, P. Exu 31/P. Exu 35, and P. Exu 40/P. Exu 44, were closely related. These strains only differed at one to three loci. The two epizootic strains unaffiliated with cluster A grouped in different clusters: $\mathrm{B}_{2}$ (P. Exu 42) and C (P. Exu 47) (Figure 4).

\section{DISCUSSION}

Studies on the molecular epidemiology of $Y$. pestis have been hampered due to the great similarity of isolates. However, new molecular approaches have uncovered a relatively high level of genetic diversity. The global expansion of $Y$. pestis strains from central Asian plague foci was demonstrated by Li et al. (2009) with an MLVA of 25 VNTRs. MLVA was also successfully employed to study the maintenance and spread of Y. pestis in Madagascar (Vogler et al., 2011).

In the present study, a phylogenetic analysis based on 12 VNTRs was performed on $Y$. pestis strains from two different epidemiological events in two northeastern Brazilian plague areas: an epizootic in Chapada do Araripe, Pernambuco State (de Almeida et al., 1985) and an outbreak in Planalto da Borborema, Paraíba State (de Almeida et al., 1989).

In Pernambuco State, plague was registered for the first time in 1902 on the docks of Recife, the capital city. Plague is suspected of spreading via transportation of cargo and reached Chapada do Araripe in 1919, establishing one of the most active plague foci in Brazil (WHO, 1965; Baltazard, 2004). MLVA performed on 20 strains from an epizootic in Sítio Alagoinha, a small property located in Chapada do Araripe in 1967, allowed us to distinguish two subgroups (Figure 4). Most of the strains (75\%) grouped in subgroup $\mathrm{A}_{1}$, suggesting that they were all from a single "epizootic path". This subgroup assembled strains from the wild rodent, $N$. lasiurus, and its fleas (P. b. jordani). This subgroup also included strains from the commensal rat ( $R$. rattus) and the rat flea, $X$. cheopis, collected on the ground inside the houses.

After studies on the ecology of plague in the Chapada do Araripe focus, Baltazard (2004) concluded that $Y$. pestis progresses as individual "epizootic paths". These "paths" wind their way randomly in short incursions through the fields, exterminating the populations of afflicted rodents. Given the extreme sedentary nature of rodents and the small radius of their excursions, such "epizootic paths" progress slowly and cover very small areas. Each "path" winds its way through the fields autonomously. This behavior could explain the presence of a dominant MLVA genotype among different species of rodents and fleas from the epizootic in a small area. 
According to Baltazard (2004), the field rodent N. lasiurus and its fleas (Polygenis spp) are by far the most frequently infected species in the wild and play an important role in plague amplification. However, other species of rodents and fleas are also involved. Our results provide further evidence of $Y$. pestis interchange between wild rodent hosts and the commensal rat and their fleas in Brazilian foci.

In Paraíba State, plague was first registered in 1912. In September 1986, after a quiescent period of 10 years, an outbreak emerged. This outbreak spread quickly throughout 41 municipalities and affected humans and several species of rodents from many localities on the Planalto da Borborema (de Almeida et al., 1989). MLVA distinguished three subgroups ( $\mathrm{B}_{1}, \mathrm{~B}_{2}$ and $\mathrm{B}_{3}$ ) among the outbreak isolates analyzed. Thus, it appears that distinct $Y$. pestis populations (the multiple "epizootic paths") circulated in the area during the outbreak. According to Baltazard (2004), when the rodent population density is high, each "epizootic path" extends and crosses and crisscrosses the other "paths". This pattern continues until the rodent population climaxes. The epizootic can then spread over vast areas, reaching all species, and then human cases arise. Most of the outbreak strains (41.2\%) clustered in subgroup $\mathrm{B}_{3}$ and one human strain (P. PB 862) clustered with two $N$. lasiurus strains isolated from the same locality.

These different $Y$. pestis populations described in the present study may reflect the microevolution of the invading strain due to environmental pressure experienced while spreading inland from the coast. Alternatively, the strains could be inaccurate representatives of the original isolates, and these differences could be due to handling procedures during the prolonged storage of the strains in the laboratory since their isolation (Revazishvili et al., 2008; Vogler et al., 2011). Leal et al. (2000) and Leal-Balbino et al. (2004, 2006) observed spontaneous changes in the genome (both plasmid and chromosomal) of Brazilian Y. pestis following prolonged storage and laboratory handling. However, analysis of the 12 VNTR loci in the three parental strains and their derived cultures did not reveal changes in the studied regions, confirming their stability as previously observed (Adair et al., 2000; Keim et al., 2000). Furthermore, according to Vogler et al. (2011), the rate of VNTR evolution in the laboratory, even with passaging, should be much slower than in nature. While these isolates may not be exactly the same as when they were first isolated, they should be quite similar to the original isolates.

In Brazil, all the plague foci have been mostly dormant since the mid-1980s. The occurrence of new cases has been rare, and the last cases were in 1997 and 2005 in Ceará. However, serological surveys continuously detect plague antibodies in sentinel animals in plague areas (Leal and Almeida, 1999; Aragão et al., 2009), suggesting that these plague foci remain active and may reappear (Duplantier et al., 2005; Gage and Kosoy, 2005; Stenseth et al., 2008; WHO, 2006, 2009).

Brazilian plague foci are autonomous, i.e., totally independent of each other, and there is no connection between them. They have different epidemiological characteristics and patterns of disease manifestations in the human population. The foci have alternating periods of high incidence followed by other periods of low incidence or even the absence of cases (Baltazard, 2004; Giles et al., 2011). The presence of similar genotypes separated by long geographical and temporal distances as in the $\mathrm{A}_{2}$ and $\mathrm{B}_{2}$ subgroups remains to be explained.

Further studies with all 43 VNTR markers previously described for $Y$. pestis (Adair et al., 2000; Klevytska et al., 2001; Girard et al., 2004) should contribute to a better understanding of the changes that have occurred in the Y. pestis genome over time in the Brazilian foci. The study of a larger number of strains from all the known Brazilian foci and strains isolated in 
different epidemiological periods may reveal the dispersion of the different $Y$. pestis "paths".

In conclusion, MLVA based on 12 VNTRs revealed that $Y$. pestis populations in Brazil are not monomorphic and demonstrated intraspecific genetic diversity among Brazilian plague strains. This study establishes a relative correlation for the first time among genetic groups based on the temporal and geographic origin of the Brazilian isolates. The knowledge of the characteristics of local strains will be very helpful in elucidating the origin of new strains, if by emergence of natural foci, reemergence or coming from other countries.

\section{ACKNOWLEDGMENTS}

Research supported by CNPq (Conselho Nacional de Desenvolvimento Científico e Tecnológico; Edital MCT/CNPq 14/2009 Process \#482694/2009-2) and SRP/CPqAMFIOCRUZ. We thank Silvana Santos from the Department of Microbiology of CPqAM for technical assistance.

\section{REFERENCES}

Achtman M, Morelli G, Zhu P, Wirth T, et al. (2004). Microevolution and history of the plague bacillus, Yersinia pestis. Proc. Natl. Acad. Sci. U. S. A. 101: 17837-17842.

Adair DM, Worsham PL, Hill KK, Klevytska AM, et al. (2000). Diversity in a variable-number tandem repeat from Yersinia pestis. J. Clin. Microbiol. 38: 1516-1519.

Altschul SF, Madden TL, Schaffer AA, Zhang J, et al. (1997). Gapped BLAST and PSI-BLAST: a new generation of protein database search programs. Nucleic Acids Res. 25: 3389-3402.

Aragão AI, Pinheiro KMA, Seoane ACM and Tavares C (2009). Prevalência de anticorpos contra Yersinia pestis em carnívoros domésticos nos focos pestosos, do Estado do Ceará. Rev. Soc. Bras. Med. Trop. 42: 711-715.

Baltazard M (1968). Viagem de estudo ao Brasil para a organização de um projeto de pesquisas sobre a peste. Rev. Bras. Malariol. Doenças Trop. 20: 335-366.

Baltazard M (2004). Recherches sur la Peste au Brésil. 4e Rapport (Mai 1970) - Progress Report. Bull. Soc. Pathol. Exot. 97 (Suppl): 93-117.

Benson G (1999). Tandem repeats finder: a program to analyze DNA sequences. Nucleic Acids Res. 27: 573-580.

de Almeida AM, Brasil DP, de Carvalho FG and de Almeida CR (1985). Isolation of Yersinia pestis in plague foci in northeast Brazil from 1966 to 1982. Rev. Inst. Med. Trop. Sao Paulo 27: 207-218.

de Almeida AM, Brasil DP, Leal NC, de Melo ME, et al. (1989). Bacteriologic and serologic studies of an outbreak of plague in the State of Paraiba, Brazil. Mem. Inst. Oswaldo Cruz 84: 249-256.

Duplantier J, Duchemin J, Chanteau S and Carniel E (2005). From the recent lessons of the Malagasy foci towards a global understanding of the factors involved in plague reemergence. Vet. Res. 36: 437-453.

Gage KL and Kosoy MY (2005). Natural history of plague: perspectives from more than a century of research. Annu. Rev. Entomol. 50: 505-528.

Giles J, Peterson AT and Almeida A (2011). Ecology and geography of plague transmission areas in northeastern Brazil. PLoS Negl. Trop. Dis. 5: e925.

Girard JM, Wagner DM, Vogler AJ, Keys C, et al. (2004). Differential plague-transmission dynamics determine Yersinia pestis population genetic structure on local, regional, and global scales. Proc. Natl. Acad. Sci. U. S. A. 101: 84088413.

Hall TA (1999). Bioedit a user-friendly biological sequence alignment editor and analysis program for Windows 95/98/ NT. Nucleic Acids Symp. Ser. 41: 95-98.

Hunter PR and Gaston MA (1988). Numerical index of the discriminatory ability of typing systems: an application of Simpson's index of diversity. J. Clin. Microbiol. 26: 2465-2466.

Karimi Y (1978). Rapid laboratory diagnosis of plague. Bull. Soc. Pathol. Exot. Filiales 71: 45-48.

Keim P, Price LB, Klevytska AM, Smith KL, et al. (2000). Multiple-locus variable-number tandem repeat analysis reveals genetic relationships within Bacillus anthracis. J. Bacteriol. 182: 2928-2936.

Klevytska AM, Price LB, Schupp JM, Worsham PL, et al. (2001). Identification and characterization of variable-number tandem repeats in the Yersinia pestis genome. J. Clin. Microbiol. 39: 3179-3185. 
Le Flèche P, Hauck Y, Onteniente L, Prieur A, et al. (2001). A tandem repeats database for bacterial genomes: application to the genotyping of Yersinia pestis and Bacillus anthracis. BMC Microbiol. 1: 2.

Leal-Balbino TC, Leal NC, Lopes CV and Almeida AM (2004). Differences in the stability of the plasmids of Yersinia pestis cultures in vitro: impact on virulence. Mem. Inst. Oswaldo Cruz. 99: 727-732.

Leal-Balbino TC, Leal NC, Nascimento MGM and Oliveira MBM (2006). The pgm locus and pigmentation phenotype in Yersinia pestis. Genet. Mol. Biol. 29: 126-131.

Leal NC and Almeida AM (1999). Diagnosis of plague and identification of virulence markers in Yersinia pestis by multiplex-PCR. Rev. Inst. Med. Trop. Sao Paulo 41: 339-342.

Leal NC, Sobreira M, Leal TCA and Almeida AMP (2000). Homology among extra-cryptic DNA bands and the typical plasmids in Brazilian Yersinia pestis. Braz. J. Microbiol. 31: 20-24.

Li Y, Cui Y, Hauck Y and Platonov ME (2009). Genotyping and phylogenetic analysis of Yersinia pestis by MLVA: insights into the worldwide expansion of Central Asia plague foci. PLoS One 22: e6000.

Lowell JL, Wagner DM, Atshabar B, Antolin MF, et al. (2005). Identifying sources of human exposure to plague. J. Clin. Microbiol. 43: 650-656.

Pollitzer R (1954). History and present distribution of plague in: plague. Monogr. Ser. World Health Organ 22: 11-45.

Pourcel C, Andre-Mazeaud F, Neubauer H, Ramisse F, et al. (2004). Tandem repeats analysis for the high resolution phylogenetic analysis of Yersinia pestis. BMC Microbiol. 4: 22.

Rasmussen SW (2002). SEQtools, A Software Package for Analysis of Nucleotide and Protein Sequences. Available at [http://www.seqtools.dk]. Accessed October 2, 2006.

Revazishvili T, Rajanna C, Bakanidze L, Tsertsvadze N, et al. (2008). Characterisation of Yersinia pestis isolates from natural foci of plague in the Republic of Georgia, and their relationship to Y. pestis isolates from other countries. Clin. Microbiol. Infect. 14: 429-436.

Rocha SS, Almeida AMP, Leal NC and Cordeiro MT (2009). Biological collections from Aggeu Magalhães Research Center, Oswaldo Cruz Foundation: analysis of a workshop. Rev. Patol. Trop. 38: 299-309.

Souza G, Abath F, Leal N and Farias A (2007). Development and Evaluation of a Single Tube Nested PCR Based Approach (STNPCR) for the Diagnosis of Plague. In: The Genus Yersinia: From Genomics to Function (Perry RD and Fetherston JD, eds.). Springer, New York, 351-359.

Stenseth NC, Atshabar BB, Begon M and Belmain SR (2008). Plague: past, present and future. PLoS Med. 5: e3.

Tamura K, Peterson D, Peterson N, Stecher G, et al. (2011). MEGA5: molecular evolutionary genetics analysis using maximum likelihood, evolutionary distance, and maximum parsimony methods. Mol. Biol. Evol. 28: 2731-2739.

Vogler AJ, Chan F, Wagner DM, Roumagnac P, et al. (2011). Phylogeography and molecular epidemiology of Yersinia pestis in Madagascar. PLoS Negl. Trop. Dis. 5: e1319.

WHO (1965). Plague in the Americas. World Health Organ. Scient. Publ. 115: 44-68.

WHO (2006). International meeting on prevention and controlling plague: the old calamity still has a future. Wkly. Epidemiol. Rec. 80: 278-284.

WHO (2009). Human plague: review of regional morbidity and mortality, 2004-2009. Wkly. Epidemiol. Rec. 85: 40-45. 